\title{
Summary of Storm-induced Dam-break Hazard of Tailings Pond
}

\author{
Qinghua Gong ${ }^{1,2}$,Jun Wang ${ }^{1,2}$,Junxiang Zhang ${ }^{3}$ \\ ${ }^{1}$ Guangzhou Institute of Geography, Guangzhou 510070, China \\ ${ }^{2}$ Guangdong Open Laboratory of Geospatial Information Technology and Application, Guangzhou 510070, China \\ ${ }^{3}$ Tourism College, Huangshan University, Huangshan 245021, China
}

\author{
暴雨诱发的矿山尾矿库溃坝事故研究综述 \\ 宫清华 ${ }^{1,2}$, 王钧 ${ }^{1,2}$, 张俊香 ${ }^{3}$ \\ ${ }^{1}$ 广州地理研究所, 广东 510070 , 中国 \\ ${ }^{2}$ 广东省地理空间信息技术与应用公共实验室, 广东 510070 , 中国 \\ ${ }^{3}$ 黄山学院旅游学院, 安徽 245021 , 中国
}

\begin{abstract}
This paper reviews the status of the tailings dam safety and flood control study. The current tailings security research mainly comprising security monitoring and early warning, failure mechanism, break model, the risk of dam failure and emergency decision-making. This paper summarized the main achievements at home and abroad in the tailings dam safety and flood control. In terms of the research content, applications and research means of the existing tailings dam accident level and future development trends were analyzed. In the future with the development of big data technology, networking technology, the tailings dam break accident monitoring and early warning of new information technologies, accident mechanism, risk analysis and emergency predict will be the future tailings research projects direction. Keywords: rain, tailings pond, dam-break, review
\end{abstract}

\section{摘要}

从尾矿库安全监测预警、溃坝机理、溃坝模型、溃坝 风险及应急决策等方面对尾矿库防汛安全与溃坝事故 研究情况进行了详细回顾, 概括总结了国内外在尾矿 库防汛安全与溃坝事故方面所取得的主要成果, 并从 研究内容、应用需求及研究手段等方面对尾矿库溃坝 事故的现有水平及未来发展动向进行了分析, 认为今 后随着低空遥感技术、物联网技术、大数据技术的发 展, 基于新的信息技术的尾矿库溃坝事故监测预警、 事故机理、风险分析及应急预测将成为未来尾矿库专 项研究的发展方向。

关键词: 暴雨、尾矿库、溃坝、综述
1. 引言

尾矿库是一种特殊的工业建筑物, 是指通过筑坝 拦截谷口或围地建造而成的, 用以堆存金属或非金属 矿山洗选矿后排出尾矿或其他工业废渣的场所 [1]。尾 矿库是矿山三大控制性工程之一, 其运行好坏不仅关 系到矿山企业的经济效益, 而且与库区下游居民的生 命财产及周边环境息息相关 $[2]$ 。尾矿库是一个具有高 势能的人造泥石流危险源, 存在溃坝危险, 一旦失事, 容易造成重特大事故。据统计, 在世界上各种重大灾 害中, 尾矿库灾害仅次于地震、霍乱、洪水和氢弹爆 炸等, 一旦发生尾矿库溃坝事故, 将严重威胁下游人 民生命财产、基础设施及生态安全。

在我国矿山诸多尾矿库中, 目前处于正常运行的 不足 $70 \%$ 。有的行 $44 \%$ 的尾矿库处于险、病、超期服务 状态, 情况非常不利 [3]。各类矿山中处于险、病、超 期服务状态的尾矿库占较高比例。截止 2011 年, 广东 也有 88 座尾矿库 (不含灰渣库), 按照 “安全度” 划 分, 正常库 41 座, 病库 9 座, 安全度不明的 38 座, 属于重大危险源的尾矿库有 16 座, 如韶关大宝山铁矿、 石人嶂铇矿, 河源的大顶铁矿、潮州市的立源锡矿等 矿山的尾矿库, 大的近千万立方米, 小的也有几十万 立方的尾砂堆积量。2010 年 9 月 21 日, 因台风 “凡 亚比” 引发强降雨导致信宜紫金矿业有限公司所属银 岩锡矿高旗岭尾矿库发生溃坝事故, 造成信宜市钱排 镇达垌、双合两村死亡 22 人, 房屋、道路、桥梁、农 田、水利灌溉设施多处被毁, 财物损失重大。

多数尾矿库很难避开生态敏感区或人口密集区, 有的 位于大江、大湖、重要水源地上游, 有的位于重要公 交设施上游, 有的在密集的居民区上游。基于溃坝后 果的严重性, 世界各国纷纷对尾矿库的安全问题均给 
Risk Analysis and Crisis Response in Big Data Era (RAC-16)

予了高度的重视。自 19 世纪以来, 各国学者分别从 尾矿库监测和预警系统开发、尾矿库溃坝事故机理、

尾矿库溃坝模型试验和数值模拟、尾矿库溃坝风险评

国际大坝委员会在 1984 年组建了国际矿山大坝 委员会和工业尾矿坝分会, 这个分会目前已经独立出 来, 针对世界日益增多的尾矿库制定了一系列安全方 面的方针, 交流各国对尾矿库安全管理工作的法规资 料和技术经验等,有利于促进尾矿库安全技术的发展。 国内在尾矿研究技术方面起步较晚, 直到 1976 年才得 到国际大坝委员会 (ICOLD) 的承认。一些专家、学者认 为尾矿坝设计较简单、工期缓慢、不利于蓄水等特点, 没有把尾矿坝作为真正的大坝来考虑, 而尾矿库属于 矿山的附属建筑物以及我们的环保意识差等原因, 尾 矿库安全性等方面存在的一些隐患一直未能引起足够 的重视。随着矿山企业的服务年限的不断延长, 尾矿 堆存体积越来越大, 其所存在的安全问题明显突出, 又因为近年来公众对环境问题越来越关注, 尾矿库所 存在的安全问题逐渐受到了有关人士的认识和重视。 尾矿库安全稳定性与许多因素有关, 如与尾砂流的运 移距离和地表干湿状态有关, 尾矿库坝体的本身物理 力学性质与区域地质构造和库区不良地质现象有关, 干滩面长度、堆坝坡度、渗透反滤以及浸润线高度会 对坝体稳定性产生影响。为了监测尾矿库的运行情况, 确定库坝的稳定性, 做出溃坝预警, 需要选取其中重 要的因素进行监测。

当前, 世界上一些发达的国家，如美国、意大利 等, 不少库坝都采用电子计算机、无线电 (或有线) 通 信、电测仪等先进技术并组成水情及坝体安全监测系 统, 已建成了现场数据采集、传输、资料分析和安全警 报的适时控制系统, 减小了数据采样或分析时间, 及时 准确地提供了水情资料, 对于科学决策提供了强有力 的依据, 我国在这方面起步较晚, 我国传统尾矿库在线 监测系统多为人工观测 [4], 是通过尾矿库周边布置的 传感器, 采集坝体浸润线、位移、滩长度、库水位等相 关数据, 并进行存储、管理和展示 [5]。国家安全生产 监督管理总局最新发布的 AQ 2030-2010《尾矿库安 全监测技术规范》, 规定了尾矿库安全监测的主要内容 包括坝体位移、渗流、干滩、库水位和降水量。传统 的监测系统因自身安全性不高、可靠性不强, 易遭受恶 劣环境影响, 监测手段严重制约着我国尾矿库安全监 测技术的进展和应用。针对这些问题, 近年来许多学者 将光纤传输 $[6]$ 、无线网络通讯技术 $[7]$ 、遥测监控技 术等手段引入到尾矿库安全监测中, 通过 (光纤传输无 线对等网络技术、无线通讯化、本地化供电模块、提 高元器件防护等) 优化和坚固化监测系统的关键环节、 专用应急指挥软件等, 构建的尾矿库安全监测体系。按
估、尾矿库溃坝应急决策等方面开展研究。

\section{2. 尾矿库安全监测预警研究}

照监测数据采集方式可分为三类, 即集中式、混合式 和分布式。目前应用最为广泛的还是分布式安全监测 系统, 它适合于对多分机, 多测点的大型尾矿库进行 监测, 在数据精确性、可靠性、实用性等方面。此外, 随着 $3 \mathrm{~S}$ 技术的发展, 利用遥感技术和全球定位系统对 尾矿库基础数据进行采集、存储、处理, 建立数据库等, 实现对信息的查询检索、综合分析、动态管理、信息 输出等功能, 也为尾矿库监测提供了新的数据采集途 径 [8] [9]。由于先进的信号传输、传感器、网络、空 间信息技术、遥测技术和软件技术的不断发展, 使得 尾矿库安全监测技术近年来在我国得到长足的进步和 极大的发展空间。

尾矿库溃坝预警是通过建立尾矿库安全预警指标 体系, 基于尾矿库安全现场监测数据, 结合指标综合 分析、事故机理分析、风险分析等模型和方法, 对尾 矿库的安全性能等预报危险程度、发布警情的过程。 尾矿库溃坝预警的核心是预警指标体系和预警模型部 分。目前国内外对尾矿库预警进行了一系列的研究, 国尾矿库预警的研究主要集中于尾矿库溃坝对环境的 区域性影响, 一般主要是针对尾矿库溃坝事故发生后 有毒有害物质如铀、氧化物和寓含金属的液态尾矿对 人类生命财产和生态环境的影响进行的研究。在国内 尾矿库的预警可分为两种, 一种是基于尾矿库监测数 据的监测预警, 其过程为通过分析、解读各项监测数据, 做出各级单项预警, 同时, 根据坝体变形、浸润线埋深、 干滩指标、库水位等监测数据的变化, 综合分析坝体的 安全稳定性, 给出预报结果。如蒋卫东等 [10]在德兴 铜矿 2 号尾矿库建立了安全自动监测系统。杨永生 [3] 在德兴铜矿建立了尾矿库水情预警预报系统, 对洪水 实现自动监测。谢振华等 [12]利用 RBF 神经网络方法, 对尾矿坝监测数据分析进行了研究。另一种是, 基于 预警指标体系和预警模型的区域预警。如谢旭阳从尾 矿库本身安全、地质条件、气象条件、人为因素等 4 个方面建立了尾矿库预警指标体系, 选取尾矿库危险 等级、地形坡度、地质构造及条件、最新日雨量、 5 日累积雨量、采矿现象、爆破现象、下游人数、下游 财产等 9 个指标作为尾矿库预警指标, 并利用支持向 量机的模型确定尾矿库的预警等级 [13-14]。

随着监测手段和尾矿库安全理论方法的发展, 今 后我国尾矿库的安全监测与预警发展方向应是建立基 于事故机理和数值模拟的尾矿库工程三维动态监测系 统, 为工程运行的判断和评价提供基础性实测数据; 建立尾矿库灾害珍断、危险性评价的智能预警系统; 
对发生事故的可能性和危害程度做出判断和评估, 对 事故发展趋势做出更科学预测。

\section{3. 暴雨型尾矿库溃坝事故机理及分析模型研究}

暴雨诱发的尾矿库溃坝过程在时空上可分为 3 个 阶段：暴雨作用下尾矿坝下开始失稳; 失稳过程中尾 矿砂和水相互作用形成具有高能量的泥石流; 高能泥 石流向下游演进。因此对于暴雨尾矿库溃坝研究机理 主要有尾矿坝坝体失稳和泥石流的演进两方面内容。

在对尾矿坝失稳研究中, 包括了尾矿坝失稳原因 和机理、尾矿坝失稳计算模型等。暴雨诱发的尾矿库 溃坝失稳的原因是是暴雨导致渗流场和应力场变化诱 发尾矿坝失稳导致溃坝, 或者是暴雨诱发洪水, 尾矿 库遭遇洪水时, 若防洪、排洪能力不足等问题, 容易 造成尾矿坝洪水漫顶, 导致尾矿库溃坝。对于尾矿库 溃坝事故机理及坝体稳定性的研究, 国内外学者采用 的研究方法主要包括理论计算、数值模拟和模型试验 等方面。在机制方面, Briaud [15]等通过试验发现, 无隸性土抗冲流速和平均粒径在对数坐标下呈线性关 系。Hancok [16] 提出了土体侵蚀度与流量、陡坎高 度及土体参数等的相关关系; 南京水利科学研究院就 溃口发展、溃口流量变化及下游洪水演进过程等问题 开展了大量的现场试验和室内模型试验研究, 已经分 别就漫顶溃坝问题和管涌溃坝问题进行了大量室内模 型试验和现场试验, 现场试验模型最高达 $9.7 \mathrm{~m}$ 。在尾 矿库渗流及模拟试验研究中提出的空间渗流数学模型 中参数单元, 具有较高精确度, 基本上能反映多层次 非均质尾矿坝的各向异性, 适用于待建尾矿库的渗流 预测和已有病、险库的渗流安全分析。

目前对尾矿库溃坝泥石流的演进主要包括模型 试验法、经验公式法和数值模拟的方法。陶东良 [17] 采用模型试验研究了某钼矿尾矿库洪水漫顶溃坝过程, 分析了洪水漫顶过程中尾矿坝的冲蚀渐近失稳过程, 给出了溃坝过程的特征时间点, 分析了不同坡比尾矿 库洪水漫顶下溃坝模式的异同。张兴凯等 [18] 基于 非恒定水流泥沙非平衡非饱和冲刷机理, 根据模型相 似理论和溃决侵蚀模型原理, 采用模型试验模拟尾矿 库洪水漫顶溃坝过程, 研究了尾矿库洪水漫顶溃坝过 程中坝体位移、浸润线高度、溃口最大流速和溃口的 演化规律, 认为尾矿库洪水漫顶溃坝位移与坝体饱和 程度有关, 坝体浸润线越高, 尾矿库溃坝时滑动位移 越大, 溃口破坏程度取决于溢流对坝体的冲刷侵蚀作 用。尾矿库溃坝泥石流的演进经验公式主要借鉴水库 溃坝后泥石流的演进, 典型的计算过程一般包括: 确 定尾矿库的总泄砂量[19-20]、尾矿库溃口的宽度 [21]、 坝址最大砂流量 [22]、坝址流量过程线 [22-23]、泥
石流演进参数计算 $[24]$ 。数值模拟法是借助参数数值 和数理模型, 对尾矿库泥石流演进进行模拟。廖威林 [25] 基于有限体积法的平面二维溃坝水流精确模拟 的基础上, 耦合非恒定流输沙的计算, 考虑了悬移质 和推移质作用下的矿砂淤积。李全明等 [26] 在建立 溃坝非恒定水沙模型的基础上, 采用数值方法预测和 模拟尾矿坝溃坝后下游洪水行进过程及矿砂淤积过程。 郭天勇 [27]采用滑动模型计算尾矿库溃坝滑移距离。 刘洋 [28]对尾矿库溃坝泥石流的演进规律进行三维数 值模拟。

虽然尾矿库溃坝后泥石流演进研究逐渐成为学术 界的热点, 但目前计算方法中, 尾矿库溃坝范围的确 定基本上都是依靠假设, 尾矿库溃口的大小仍然借用 水库溃坝的溃口经验公式, 尾矿库的溃坝过程基本都 是假设一次全溃, 这都会对计算结果产生较大的影响, 尚需要进一步的深入研究。

综上, 尾矿库溃坝事故机理研究多从理论分析和 现场调查方面来探讨, 而物理模型试验方法却主要在 研究尾矿库稳定性方面采用, 尾矿库溃坝计算模型还 不完善 [29], 需要借助土力学、水力学和泥沙动力学 等学科的交叉进一步研究。

\section{4. 尾矿库溃坝风险评价研究}

尾矿库风险评价是保障尾矿库运行安全的重要 部分, 有助于指导设计、施工和尾矿库安全管理。风 险管理是使保障工程生产运营安全的前提 [30]。目前 尾矿库溃坝风险评估的通用方法, 是以尾矿库固有致 灾强度的自然属性如尾矿坝边坡稳定安全系数等作为 孕灾环境指标、以社会经济因素 (如尾矿库下游人口 分布、社会经济发展水平和资源环境条件等) 为承灾 环境易损性或脆弱性指标, 综合建立风险评估指标体 系, 在通过风险评估模型, 建立风险评价体系, 对尾 矿库溃坝风险等级做出评定。1980 年初, 美国发表了 不少有关尾矿库坝体溃坝风险分析方法和原理的文章, 推动风险分析技术在尾矿库溃坝事故中应用的发展。 美国垦务局在相对风险指数法的基础上，采用了现场 评分的方法来评价溃坝的风险, 并将根据险情将风险 化成了级。在澳大利亚, 安全评价人员通常是将风险 定性和定量分析相结合来对尾矿库的安全进行评价。 对尾矿库常采用的分析方法是使用事件树或者故障树。 然后列出尾矿库所存在的安全隐患, 接着依靠专家经 验或者对隐患的发生进行可能性概率分析, 对尾矿坝 的风险进行最终的评价。2000 年在北京召开第二十届 国际大坝会议, 第一次把大坝风险分析作为会议主题。 标志着水库大坝工程风险分析已经发展成为体系较完 整的决策工具, 针对尾矿库溃坝风险评价的方法和模 


\section{Risk Analysis and Crisis Response in Big Data Era (RAC-16)}

型, 国内外学者开展了很多理论和实践方面的研究, 其评价方法主要有层次分析法、模糊层次综合分析法、 人工神经网络法、可靠度理论法、模糊数学综合评价 法、鱼刺图法、Monte-Carlo 模型等。谭星宇 [31]、 李全明 [32] 用相关性分析, 将与尾矿坝安全有关的参 数和设计指标进行归类, 建立了相互独立指标的尾矿 库溃坝风险指标体系, 并应用模糊理论建立了尾矿库 溃坝风险评价模型。吴令 [34]用鱼刺图法评价得到了 尾矿库溃坝事故的危险程度。梅国栋 [33]运用层次分 析方法, 建立了尾矿库溃坝灾害脆弱性评估指标体系, 进而完成尾矿库溃坝灾害脆弱性风险评估。在对尾矿 库溃坝风险进行评价时, 目前较多运用的是将 A H P 和模糊理论或其他相关理论相结合的方法。以上常用 的方法是定量和半定量的方法, 评价结果的主观性较 强, 针对目前我国尾矿库安全评价中存在的不足, 梅 国栋 [32] 基于风险评估理论和定量评价方法，采用 Monte-Carlo 模型计算尾矿坝溃坝失效概率、有限差 分法计算溃坝淹没范围, 定量计算尾矿库溃坝后生命 损失、财政损失和环境损失, 最终确定尾矿库溃坝风 险度。

综上可见, 近年来, 我国的尾矿库溃坝风险评价 理论和方法研究得到了长足的长进, 但由于尾矿坝溃 坝灾害的严重性及溃坝过程的复杂性, 对溃坝风险评 价模型及溃坝过程的研究仍将是一个重要的课题。今 后, 对尾矿库溃坝风险评价的研究仍将会以探索更加 实用、简便、准确的风险评价方法和模型为主。

\section{5. 尾矿库溃坝事故应急决策研究}

发生尾矿库溃坝事故时, 当地安全生产监督管理 部门和人民政府根据尾矿库溃坝的风险情况，对事故 应急救援、转移疏散等进行应急决策, 应急决策的目 的是事故发生前, 预测潜在的重大危险、事故类型、 事故发生的地点, 事故发生后及时有效的指挥救援单 位进行救援行动。尾矿库溃坝预警信息的辨识、获取 和分析, 是有效防止尾矿库溃坝的关键技术 [36]。在 当前的研究领域, 主要针对公共安全突发事故的应急 决策, 暂未发现专门针对尾矿库溃坝事故的应急技术 研究。

国外应急决策体系针对可能的重大事故或灾害预 先制定的有关计划或方案。在当前的应急决策研究领 域, 主要集中于应急决策系统的研究。国际上在 20 世纪 80 年代以来逐步建立了若干个以灾害信息服务、 灾害应急事务处理为目标的灾害信息系统, 主要有: 由加拿大应急管理署主持的全球危机和应急管理网络, 由美国联邦应急管理局主持的全球应急管理与紧急响 应联系系统, 由联合国国际减灾十年办公室主持开发
的国际灾害信息资源网络, 由挪威、法国、芬兰、丹 麦四国共同开发的模块化紧急管理系统, 以及日本灾 害应变系统等。在我国, 针对一些重要灾种 (如地质 灾害等) 进行监测、预报、应急响应决策分析的专题系 统正在或已经建成 [37]。随着研究的深入以及技术方 法的不断创新和进步, 全国性综合性的灾害信息系将 在不久建成并投入使用。虽然在应急决策研究和工作 中取得了一定进展, 但在开展过程中仍然有许多关键 技术问题亟需解决, 如集成化、分布式等, 其中最重 要的是如何从工作需求出发, 有效实现系统的全面业 务化, 充分发挥现代信息技术、计算机技术、空间科 学技术等在灾害管理工作中应具有的重要作用。

目前对尾矿库溃坝事故缺乏有效的应急机制, 在 尾矿库选址的防汛安全的空间决策和溃坝应急空间决 策方面缺少有效的技术和手段。在溃坝事故应急响应 中暴露出两方面的问题: 一方面, 对尾矿库的形成机 理等问题研究基础薄弱, 事故应急管理缺乏科学依据; 另一方面, 不能快速地对险情空间布置做出的科学判 断。随着地球空间信息科学的发展, 地球空间信息学 已经成为突发事件应急管理理论支撑的重要组成部分。 由于突发事故往往是典型的空间分布对象, 具有典型 的空间分布特征, 因此突发公共安全事故过程对空间 信息 (包括几何与属性) 的综合性、连续性获取具有迫 切需求, 同时需要把其它相关的统计、文字等数据展 布在空间图层上, 进而开展一系列计算、分析、评价 与应用工作 (如事故风险评价、损失评估、事故信息服 务等)。

\section{6. 未来研究趋势}

从国内外发展趋势看, 空间信息技术正朝着有利 于提高突发事件应急管理工作水平的方向发展, 三维 激光扫描技术等具有快速反应、快速测绘、快速成果 提取等特点, 在突发事件应急空间决策方面有很强的 技术优势。因此, 对于尾矿库溃坝应急决策未来的趋 势为, 借助空间信息技术在溃坝事故事件应急管理与 应急响应业务工作中将得到深入应用 [38], 建立一体 化、业务化运行的应急响应决策支持系统。随着三维 空间信息技术、大数据技术、物联网技术的发展, 基 于新的信息技术的矿山尾矿库溃坝事故研究成为未来 的发展方向。矿山尾矿库危险源的空间分布、事故的 危害范围和事故应急救援路线等都具有明显的地理空 间特征, 因此充分发挥空间信息技术在对空间数据的 采集、存储、管理、运算、分析、显示和描述等方面 的优势, 研发危险源识别与分级, 事故危害范围和事 故规模预测、事故损失预测和应急空间目标和应急调 度路线选择等关键技术成为防灾减灾的需求和发展方 
向。

\section{7. 结论}

本文对暴雨诱发的矿山尾矿库溃坝事故研究综述 从尾矿库安全监测预警、溃坝机理、溃坝模型、溃坝 风险及应急决策等方面对尾矿库防汛安全与溃坝事故 研究情况进行了详细回顾。在尾矿库的专项分析研究 中, 对于尾矿库坝体的稳定性评价及尾矿库坝体渗流 稳定性评价方面做了大量的工作, 但尾矿库的综合研 究、尾矿库溃坝事故机理研究, 防汛安全空间设计和 溃坝应急决策方面尚为不足。为有效防止及应对尾矿 库溃坝事故, 研究溃坝事故形成机理和溃坝应急空间 决策模型, 为尾矿库选址和溃坝应急决策提供有效的 科学依据是一项刻不容缓的任务。极有必要进一步开 展溃坝研究, 尤其要大力开展溃坝模型试验研究, 从 溃坝形成机理入手, 进而对溃坝洪水、泥沙运动等其 它相关内容开展深入研究, 从而为工程设计施工提供 直接依据。随着三维空间信息技术、大数据技术、物 联网技术的发展, 基于新的信息技术的矿山尾矿库溃 坝事故研究成为未来的发展方向。

\section{Acknowledgements}

This study was supported by Science and Technology

Planning Project of Guangdong Province, China (No.

2012A030200010, No.2013B031500007, and 2014A020218013)

\section{致谢}

本研究得到广东省科技计划项目 (2012A030200010, 2013B031500007，2014A020218013）联合资助。

\section{参考文献}

[1] 李全明,王云海,张兴凯,赵军. 尾矿库溃坝灾害因 素分析及风险指标体系研究.中国安全生产科学 技术,2008,03:50-53.

[2] 陈殿强,何峰,王来贵.风城市某尾矿库溃坝数值计 算.金属矿山,2012(10):74-80.

[3] 徐宏达.我国尾矿库病害事故统计分析. 工业建 筑.2003.31(1).

[4] 洪振川.尾矿库安全监测预警系统在南山矿尾矿 库的应用.现代矿业,2010,06:129-130+138.

[5] 李青石,李庶林,陈际经.试论尾矿库安全监测的现 状及前景. 中国地质灾害与防治学 报,2011,01:99-106.
[6] 许同乐,郎学政,裴新才,薛丽贤.基于光纤传输的 尾矿库安全监测预警系统研究. 黄 金,2011,07:43-47.

[7] 马文可,魏诺,付勇. 基于无线传感网络的尾矿库 安全监测预警系统的设计与实现. 山东科 学,2011,03:79-82.

[8] 吕杰.尾矿库遥感监测技术研究与应用.中国地质 大学 (北京),2014.

[9] 梅国栋, 尾矿库溃坝机理及在线监测预警方法研 究[D].北京科技大学,2015.

[10] 蒋卫东, 李夕兵, 吴大志,赵国彦德兴铜矿 2 号尾矿 坝的安全自动监测系统. 矿业研究与开 发,2003,01:33-34+44.

[11] 杨永生. 尾矿库水情预警预报系统. 矿业快 报,2006(6):24〜 27.

[12] 谢振华, 陈庆. 尾矿坝监测数据分析的 RBF 神经网 络方法.金属矿山,2006(10):69-71。

[13] 谢旭阳,江田汉,王云海,张兴凯. 基于支持向量机 的尾矿库灾害区域预警.中国安全生产科学技 术,2008,04:17-21.

[14] 谢旭阳,王云海,张兴凯, 李全明.尾矿库区域预警 指标体系的建立. 中国安全科学学 报,2008,05:167-171.

[15] Briaud. J. L. Case Histories in soil and rock erosion: Woodrow Wilson Bridge, Brazos River Meander, Normandy Cliffs,andNew Orleans Levees Journal of Geotechnical and Geoenvironmental Engineering, 2008, 134(10): $1424-1447$.

[16] Hancock G R. The use of landscape evolution models in mining rehabilitation design. Environmental Geology, 2004, 46( 5) : 578 -583 .

[17] 陶东良. 某钼矿尾矿库洪水溃坝模型试验研究. 工 程设计与研究,2011,130: 18-24.

[18] 张兴凯, 孙恩吉, 李仲学. 尾矿库洪水漫顶溃坝演 化规律试验研究.中国安全科学学报,2011,21 (7): 118-124.

[19] 陈青生,孙建华.矿山尾矿库溃坝砂流的计算模拟. 河海大学学报, 1995，23（5): 99-105.

[20] 袁兵, 王飞跃, 金永健. 尾矿坝溃坝模型研究及应 用.中国安全科学学报，2008，18（4): 169-172.

[21] 李炜.水力计算手册. 北京: 中国水利水电出版社, 2006.

[22] 王又武, 袁平, 陈珂佳. 尾矿库溃坝有关问题探讨 [J].工程建设,2009,41 (5): 35-41.

[23] 许远瑶, 乔永安. 溃坝水流计算的几个问题. 人民黄 
Risk Analysis and Crisis Response in Big Data Era (RAC-16)

河,1983（6): 30-33

[24] 康志成, 李悼芬, 马蔼乃等.中国泥石.流研究. 北京: 科学出版社,2004.

[25] 廖威林.尾矿库溃坝尾砂下泄数值模拟研究. 华南 理工大学, 2015

[26] 李全明,李玲,王云海.尾矿库溃坝淹没范围的定量 计算方法研究. 中国安全科学学报, 2011, 21 (11): 92-96.

[27] 郭天勇,武伟伟,段蔚平,杨强胜. 尾矿库溃坝滑坡 体滑移距离的研究.金属矿山,2014,12:193-197.

[28] 刘洋,齐清兰,张力霆. 尾矿库溃坝泥石流的演进 过程及防护措施研究.金属矿山,2015,12:139-143.

[29] 张力霆. 尾矿库溃坝研究综述. 水利学 报,2013,05:594-600.

[30] D. Baloi. Risk analysis techniques in construction engineering projects. Journal of Risk Analysis and Crisis Response, 2012,02: 115-123

[31] 谭星宇,谢贤平,王彦波. 基于模糊层次分析的尾 矿库溃坝风险评价。中国非金属矿工业导
刊,2014,01:55-58.

[32] 李全明,张兴凯,王云海,张丙印.尾矿库溃坝风险 指标体系及风险评价模型研究. 水利学 报,2009,08:989-994.

[33] 梅国栋. 尾矿库溃坝灾害脆弱性评估指标体系及 方法研究.中国安全生产科学技术, 2012，(12): 11-15.

[34] 吴令, 周波.鱼刺图评价法在尾矿库溃坝事故中的 应用.价值工程2012,02:280-281.

[35] 梅国栋, 吴宗之. 尾矿库溃坝风险定量评价方法探 讨.中国安全生产科学技术,2012,(02):78-82.

[36] 吴宗之,梅国栋. 尾矿库溃坝预警体系及预警方法 研究.金属矿山,2014,12:198-202.

[37] 杨义辉,冯仁俊,李明建,岳俊,宋志强.基于 GIS 的 矿井应急救援系统的研究及应用. 矿业安全与环 保,2009,S1:64-67+247.

[38] 于广明,宋传旺,潘永战,李亮, 李冉, 路世豹. 尾矿 坝安全研究的国外新进展及我国的现状和发展态 势.岩石力学与工程学报,2014,S1:3238-3248. 